\title{
DETERMINING THE COHOMOLOGICAL DIMENSION OF CERTAIN COMPACTA
}

\author{
LEONARD R. RUBIN
}

\begin{abstract}
In previous work of this author and of John J. Walsh it was shown that many of the known examples of hereditarily infinite dimensional compacta have infinite cohomological dimension. In this paper, the class of compacta whose cohomological dimension is known to be infinite will be enlarged.
\end{abstract}

1. Introduction. The results of this paper form a part of a systematic approach for calculating the cohomological dimension of compacta that are known to be hereditarily infinite dimensional. This work was begun in [Wal] and has been carried further in [Ru2, Ru3]. Spaces that are hereditarily infinite dimensional are important in the study of cell-like maps and specifically in the problem of whether cell-like maps can raise dimension. It is known that if there exists a compactum whose dimension is infinite but whose cohomological dimension is finite, then that compactum will be the image of a cell-like dimension raising map [Wa2]. Such a space, if strongly infinite dimensional, contains by [Ru1] a hereditarily strongly infinite dimensional closed subspace. For short expositions of the background material in this area, one should consult [GS, Ru4].

For the current paper, it is best to key in on the result Theorem 3.1 of [Wa1]; let us state it here for reference.

1.1. Theorem. Let $\left\{N_{1}, N_{2}\right\}$ be a partition of the natural numbers. For each $k \in N_{1}$, let $S_{k}$ be a separator of the kth opposite faces $A_{k}$ and $B_{k}$ of the Hilbert cube. Let $X=\bigcap\left\{S_{k} \mid k \in N_{1}\right\}$; then for each finite subset $\Lambda$ of $N_{2}$, the projection of $X$ into the $\Lambda$-coordinates is cohomologically stable. In particular, c-dim $X \geqslant \operatorname{card}\left(N_{2}\right)$.

In order to prove that c-dim $X=\infty$ it is required that there be an infinite set $N_{2}$ of natural numbers such that in the construction of the space $X$, no separators of the faces $A_{k}$ and $B_{k}\left(k \in N_{2}\right)$ of the Hilbert cube are to be used. In spite of that restriction, it was still shown in $\S 7$ of [Wa1] that most of the extant examples (which were not necessarily constructed directly in accordance with the above theorem) had infinite cohomological dimension.

Received by the editors March 14, 1986 and, in revised form, July 7, 1986.

1980 Mathematics Subject Classification (1985 Revision). Primary 55M10, 54F45.

Key words and phrases. Cohomological dimension, hereditarily infinite dimensional, essential family, cell-like mapping.

The author is a Fulbright Lecturer at the University of Zagreb while on Sabbatical leave from the University of Oklahoma. 
On the other hand, examples constructed in [Ru1] do not readily yield to this analysis. In [Po1] there are new constructions of hereditarily strongly infinite dimensional compacta as intersections of separators in the Hilbert cube, and which would appear even more difficult to analyze via 1.1. It is the purpose of this paper to obtain a theorem similar to 1.1, but which will show that all examples in [Ru1, Po1] of hereditarily strongly infinite dimensional compacta constructed as intersections of separators in the Hilbert cube have infinite cohomological dimension.

A few comments on the nature of the results in [Ru2, Ru3] are in order here. Since the results in [Ru3] on calculating cohomological dimension generalize those in [Ru2], it is sufficient to comment on [Ru3]. Foremost it must be noted that the emphasis there is on spaces which are not the intersection in $I^{\infty}$ of a collection of separators of opposite face pairs. Rather, that paper covers spaces that are related to intersections of separators with another space $Y$ lying in $I^{\infty}$, where $Y$ has a certain dimension intersection property, $\operatorname{DIP}\left(\Gamma_{1}, \Gamma_{2}\right)$. This is described in $\S 3$ of [Ru3] at the bottom of p. 223.

The Corollary to Theorem 3.2 of [Ru3] then shows that a certain class of compacta has infinite cohomological dimension. It is interesting to note, though, that a requirement similar to one in [Wa1], that an infinite number of face pairs not be separated, is also used. The author is studying this and comparing it with the present paper to see if this stipulation can be eliminated.

2. Preliminaries. Spaces are assumed metrizable if not otherwise stated. Let $\omega$ denote the natural numbers and for each $j \in \omega$, let $I_{j}=[-1,1]$. We use $I$ for $[0,1]$ and $I^{\infty}$ for $\Pi\left\{I_{j} \mid j \in \omega\right\}$, the Hilbert cube. If $\Lambda \subset \omega$, then let $I^{\Lambda}$ be the set of points $x$ in $I^{\infty}$ such that $x_{j}=0$ if $j \notin \Lambda$; but if $k \in \omega$, then $I^{k}$ will usually denote those $x$ for which $x_{j}=0$ when $j>k$. Hence $I^{k}$ is a $k$-cell and we shall use $S^{k}$ to denote $\partial I^{k+1}$.

The notations $\pi_{\Lambda}: I^{\infty} \rightarrow I^{\Lambda}$ and $\pi_{j}: I^{\infty} \rightarrow I_{j}$ will be used to denote (coordinate) projections. The $j$ th faces of the Hilbert cube are $A_{j}=\pi_{j}^{-1}(-1)$ and $B_{j}=\pi_{j}^{-1}(1)$, $j \in \omega$.

If $A, B$ are disjoint closed subsets of a space $X$, and $S$ is a closed subset of $X$ such that $X-S$ is the union of separated sets $U, V$ with $A \subset U, B \subset V$, then we call $S$ a separator of $(A, B)$ in $X$. For the notions of essential family, EilenbergMac Lane space, stable mapping, cohomologically stable mapping, one should consult [Ru2]. Other terminology not covered there can be found in [Ru3, Wa1]. Let us assume though, without loss of generality, that each Eilenberg-Mac Lane space $K(\mathbf{Z}, n)=K_{n}$ dealt with herein is an ANE for metric spaces. Also we assume that $K_{n}$ is obtained from $S^{n}$ by attaching cells of dimension $\geqslant n+2$.

For convenience, let us state Lemma 2.7 of [Ru3].

2.1. Lemma. Let $M_{0}$ be a space of dimension $m+1$, let $\left\{\left(A_{k}^{\prime}, B_{k}^{\prime}\right) \mid 1 \leqslant k \leqslant m+1\right\}$ be an essential family for $M_{0}$, and let $A=\bigcup\left\{\left(A_{k}^{\prime} \cup B_{k}^{\prime}\right) \mid 1 \leqslant k \leqslant m+1\right\}$. Suppose $F: A \rightarrow S^{m}$ is a map such that $A_{k}^{\prime}=F^{-1}\left(A_{k} \cap S^{m}\right), B_{k}^{\prime}=F^{-1}\left(B_{k} \cap S^{m}\right), 1 \leqslant k \leqslant$ $m+1$. Then there does not exist an extension of $F$ to a map of $M_{0}$ to $K_{m}$. 
Suppose now that $0<a_{j}<1, j \in \omega$, and define $A_{j}^{*}=\pi_{j}^{-1}\left[-1,-a_{j}\right], B_{j}^{*}=$ $\pi_{j}^{-1}\left[a_{j}, 1\right]$. Let us call $\left(A_{j}^{*}, B_{j}^{*}\right)$ a thickening of the faces $\left(A_{j}, B_{j}\right)$. This terminology will be convenient for use in the next section.

3. Examples. In $\S 5$ of [Po1], the author constructed hereditarily strongly infinite dimensional compacta in $I^{\infty}$ of the form $\bigcap\left\{L_{j} \mid j \in \Gamma\right\}$ where $\Gamma \subset \omega$ is infinite and $\omega-\Gamma \neq \varnothing$. In all other respects $\Gamma$ is arbitrary. The sets $L_{j}$ are separators in $I^{\infty}$ of $\left(A_{j}, B_{j}\right)$, chosen in a very careful way. For simplicity one usually assumes $1 \notin \Gamma$, and this we do in the sequel. Examining 5.1, 5.2, 5.3 of [Po1] one can detect that the following are true.

3.1. (a) $\Gamma$ is an infinite subset of $\omega-\{1\}$ and $T$ is a totally disconnected subset of $I_{1}$.

(b) For each $j \in \Gamma, L_{j}$ is a separator of $\left(A_{j}, B_{j}\right)$ in $I^{\infty}$.

(c) There is an infinite subset $\Gamma^{*}$ of $\Gamma$ having the property that if $\sigma$ is a subset of $\Gamma^{*}$ and $M \subset \bigcap\left\{L_{j} \mid j \in \sigma\right\}$ is such that $\pi_{1}(M) \supset T$, then $\left\{\left(A_{j}^{*} \cap M, B_{j}^{*} \cap M\right) \mid j\right.$ $\in \sigma\}$ is an essential family for $M$ for some fixed thickening $\left(A_{j}^{*}, B_{j}^{*}\right)$ of the faces $\left(A_{j}, B_{j}\right), j \in \sigma$.

Pol's construction is rather efficient, and the above facts are readily obtained from it even though they were not stated as such. On the other hand, the construction of hereditarily strongly infinite dimensional compacta in [Ru1] is more complicated, and the properties of this construction are less readily discerned. Still, a careful examination of $\S 6$ thereof will show a certain similarity between it and that of [Po1].

Again the compacta are of the form $\bigcap\left\{L_{j} \mid j \in \Gamma\right\}$ with the same restriction on $\Gamma$ as above (we used $Z_{j}$ instead of $L_{j}$ there). The major difference which bears upon the present paper comes as a result of Theorem 4.9 of [Ru1] and appears in the proof of Theorem 6.2 where it is necessary to be able to select from an infinite set of indices, $\lambda^{-1}(W, n)$, in order to be sure that $\bigcap\left\{F\left(S_{m}^{\prime \prime}\right) \mid m \in \lambda^{-1}(W, n)\right\} \subset \bigcap\left\{\tilde{S}_{m} \mid m\right.$ $\left.\in \lambda^{-1}(W, n)\right\}$. If we substitute any infinite subset of $\lambda^{-1}(W, n)$ for $\lambda^{-1}(W, n)$ in the preceding, we could still achieve the stated inclusion, but the proof requires an infinite set. For this reason, the examples of [Ru1] satisfy something weaker than what is listed in 3.1, namely the following.

3.2. (a) $\Gamma$ is an infinite subset of $\omega-\{1\}$ and $T$ is a totally disconnected subset of $I_{1}$.

(b) For each $j \in \Gamma, L_{j}$ is a separator of $\left(A_{j}, B_{j}\right)$ in $I^{\infty}$.

(c) There is an infinite subset $\Gamma^{*}$ of $\Gamma$ having the property that if $\sigma$ is an infinite subset of $\Gamma^{*}$ and $M \subset \bigcap\left\{L_{j} \mid j \in \sigma\right\}$ is such that $\pi_{1}(M) \supset T$, then $\left\{\left(A_{j}^{*} \cap M, B_{j}^{*}\right.\right.$ $\cap M) \mid j \in \sigma\}$ is an essential family for $M$ for some fixed thickening $\left(A_{j}^{*}, B_{j}^{*}\right)$ of the faces $\left(A_{j}, B_{j}\right), j \in \sigma$.

3.3. Proposition. The examples of the form $\cap\left\{L_{j} \mid j \in \Gamma\right\}$ of $\S 5$ of [Po1] satisfy 3.1, and those of [Ru1] satisfy 3.2.

Although the examples in [Po1] appear to have stronger properties, we shall see that the conditions in 3.2 are sufficient to prove that any example of either type has infinite cohomological dimension.

3.4. Note. Any space of the form $\bigcap\left\{L_{j} \mid j \in \Gamma\right\}$ satisfying 3.1 also satisfies 3.2. 
4. Calculations of cohomological dimension. Here now is the main theorem of the paper.

4.1. Theorem. Any space $X=\bigcap\left\{L_{j} \mid j \in \Gamma\right\}$ where $\left\{L_{j} \mid j \in \Gamma\right\}$ and $T$ are as in 3.2 has infinite cohomological dimension.

4.2. Corollary. The hereditarily strongly infinite dimensional spaces of [Ru1] and of $\S 5$ of [Po1] have infinite cohomological dimension.

Proof. The corollary follows from 3.3, 3.4 and the theorem. To prove the theorem, let $\Lambda \subset \Gamma^{*}$ be finite. Let $I^{\Lambda}$ be denoted by $I^{m+1}$ and $S^{m}=\partial I^{m+1}$, where $m+1=\operatorname{card} \Lambda$. Let $\pi=\pi_{\Lambda}: I^{\infty} \rightarrow I^{m+1}$. There is a map $r: I^{\infty} \rightarrow I^{\infty}$ which is the identity on $\pi^{-1}\left(S^{m}\right)$ and so that $r^{-1}\left(A_{j}\right) \supset A_{j}^{*}, r^{-1}\left(B_{j}\right) \supset B_{j}^{*}$ for $j \in \Lambda$. Let $e=\pi \circ r: I^{\infty} \rightarrow I^{m+1}$. It is our aim to show that the map $e \mid X: X \rightarrow I^{m+1}$ is cohomologically stable. Suppose though that it is not; so there is a map $F: X \rightarrow K_{m}$ which agrees with $e$ on $e^{-1}\left(S^{m}\right) \cap X$. Extend $F$ to a map of $X \cup e^{-1}\left(S^{m}\right)$ to $K_{m}$ by defining $F(x)=e(x)$ for $x \in e^{-1}\left(S^{m}\right)$. Using the fact that $K_{m}$ is an ANE, extend $F$ further to an open neighborhood $U$ of $X \cup e^{-1}\left(S^{m}\right)$ in $I^{\infty}$. You can check that $F^{-1}\left(A_{j} \cap S^{m}\right) \supset A_{j}^{*}, F^{-1}\left(B_{j} \cap S^{m}\right) \supset B_{j}^{*}$ for $j \in \Lambda$. Choose $V$ to be an open neighborhood of $X \cup e^{-1}\left(S^{m}\right)$ so that $\bar{V} \subset U$.

Choose $k$ so large that any map $\pi^{*}: I^{\infty} \rightarrow I^{\infty}$ which is the identity on the first $k$ coordinates is so close to the identity that $\pi^{*}\left(X \cup e^{-1}\left(S^{m}\right)\right) \subset V$ and $\pi^{*}(V) \subset U$. Also choose $k$ so that $I^{m+1} \subset I^{k}$, i.e., so that all elements of $\Lambda$ are less than $k$. Define subsets $\Lambda_{1}=\{2, \ldots, k\} \cap(\Gamma-\Lambda)$ and $\Lambda_{2}=\{2, \ldots, k\}-\left(\Lambda \cup \Lambda_{1}\right)$ of $\{2, \ldots, k\}$. Thus $\left\{\Lambda, \Lambda_{1}, \Lambda_{2}\right\}$ is a pairwise disjoint collection of subsets of $\{2, \ldots, k\}$ whose union is $\{2, \ldots, k\}$.

There is a homotopy $h: I^{\infty} \times I \rightarrow I^{\infty}$ given by $h(x, t)=y$ where $y_{i}=x_{i}$ for $i \leqslant k$ and $y_{i}=(1-t) x_{i}$ for $i>k$. Let $h_{t}(x)$ denote $h(x, t)$. Then $h_{t}$ is an embedding for all $t_{0}<1$, and $h_{1}=\pi_{k}: I^{\infty} \rightarrow I^{k}$.

For each $j \in \Lambda_{1}$, let $L_{j}^{\prime}=L_{j} \cap I^{k}$. Let $X_{1}=\bigcap\left\{L_{j} \mid j \in \Gamma-\Lambda_{1}\right\}$ and let $X^{\prime}=$ $X_{1} \cap \cap\left\{L_{j}^{\prime} \mid j \in \Lambda_{1}\right\}$. Thus $X^{\prime} \subset X$ and by virtue of the choice of $k$ and the fact that each $h_{t}$ is the identity on the first $k$ coordinates, we see that $h\left[\left(X^{\prime} \cup e^{-1}\left(S^{m}\right)\right)\right.$ $\times I]$ is a compact subset of $V$. If $X^{\prime \prime}$ is within a sufficiently small neighborhood of $X^{\prime}$, then we also have $h\left(X^{\prime \prime} \times I\right) \subset V$. Hence it is true that if $L_{j}^{*}, j \in \Lambda_{1}$, is chosen sufficiently close to $L_{j}^{\prime}$ in $I^{\infty}$, then we will have $h\left(X^{\prime \prime} \times I\right) \subset V$ where $X^{\prime \prime}=X_{1} \cap$ $\bigcap\left\{L_{j}^{*} \mid j \in \Lambda_{1}\right\}$. We select thus a transverse collection $\left\{L_{j}^{*} \mid j \in \Lambda_{1} \cup \Lambda_{2}\right\}$ of $(k-1)$-dimensional PL manifolds in $I^{k}$ so that each $L_{j}^{*}$ is a separator of $\left(A_{j} \cap\right.$ $\left.I^{k}, B_{j} \cap I^{k}\right)$ in $I^{k}$ and for $j \in \Lambda_{1}, L_{j}^{*}$ is "close" to $L_{j}^{\prime}$. It is sufficient that these sets $L_{j}^{*}$ be polyhedra. Then $M=\bigcap\left\{L_{j}^{*} \mid j \in \Lambda_{1} \cup \Lambda_{2}\right\}$ is a polyhedron whose dimension is at most $k-\operatorname{card}\left(\Lambda_{1} \cup \Lambda_{2}\right)=m+2$, and $h\left(X^{\prime \prime} \times I\right) \subset V$ where $X^{\prime \prime}=X_{1}$ $\cap M$.

Suppose $0 \leqslant t<1$. Then the embedding $h_{t}$ is the identity on $I^{k}$; thus

$$
h_{t}\left(X^{\prime \prime}\right)=h_{t}\left(X_{1}\right) \cap M=\bigcap\left\{h_{t}\left(L_{j}\right) \mid j \in \Gamma-\Lambda_{1}\right\} \cap M .
$$

For each $i>k$, let $J_{i}=[t-1,1-t]$, let $Q_{k}=\Pi\left\{J_{i} \mid i>k\right\}$ and choose $t$ so close to 1 that $K=h_{t}\left(X_{1}\right) \cap \cap\left\{L_{j}^{*} \times Q_{k} \mid j \in \Lambda_{1} \cup \Lambda_{2}\right\} \subset V$. 
Define $\tilde{L}_{j}$ to be $L_{j}^{*} \times Q_{k}, j \in \Lambda_{1} \cup \Lambda_{2}$. Note that $\tilde{L}_{j}$ is a separator of $\left(A_{j} \cap\left(I^{k} \times Q_{k}\right), \quad B_{j} \cap\left(I^{k} \times Q_{k}\right)\right)$ in $I^{k} \times Q_{k}$, the latter being a copy of the Hilbert cube. Also, $h_{t}\left(A_{j}\right)=A_{j} \cap\left(I^{k} \times Q_{k}\right)$ and $h_{t}\left(B_{j}\right)=B_{j} \cap\left(I^{k} \times Q_{k}\right)$ for $j \in \Lambda_{1} \cup \Lambda_{2}$. On the other hand, for $j \in \Gamma-\Lambda_{1}, h_{t}\left(L_{j}\right)$ is a separator of $\left(h_{t}\left(A_{j}\right), h_{t}\left(B_{j}\right)\right)$ in $I^{k} \times Q_{k}=h_{t}\left(I^{\infty}\right)$. It folows by 5.5, 5.2 of [RSW] that $K=$ $\bigcap\left\{h_{t}\left(L_{j}\right) \mid j \in \Gamma-\Lambda_{1}\right\} \cap \cap\left\{\tilde{L}_{j} \mid j \in \Lambda_{1} \cup \Lambda_{2}\right\}$ contains a continuum from $A_{1}$ to $B_{1}$ in $I^{k} \times Q_{k}$ since $1 \notin \Gamma \cup \Lambda_{2}=\left(\Gamma-\Lambda_{1}\right) \cup \Lambda_{1} \cup \Lambda_{2}$.

From the preceding we see that $h_{t}^{-1}(K)$ contains a continuum from $A_{1}$ to $B_{1}$. Also $h_{t}^{-1}(K) \subset \bigcap\left\{L_{j} \mid j \in \Gamma-\Lambda_{1}\right\}$, and $\Gamma-\Lambda_{1}$ contains the subset $\Lambda$ of $\Gamma^{*}$ as well as an infinite subset of $\Gamma^{*}$. For each $x \in T$, where $T \subset I_{1}$ is the totally disconnected set of 3.2(a), choose a point $y_{x} \in \pi_{1}^{-1}(x) \cap h_{t}^{-1}(K)$ and let $\Delta=$ $\left\{y_{x} \mid x \in T\right\}$. Hence by $3.2(\mathrm{c}), \Delta$ has an infinite essential family; but for our purposes we only use the information that $\left\{\left(A_{i}^{*} \cap \Delta, B_{i}^{*} \cap \Delta\right) \mid i \in \Lambda\right\}$ is an essential family for $\Delta$. Since $h_{t}$ does not change $\Lambda$-coordinates or the first coordinate, then $\left\{\left(A_{i}^{*} \cap h_{t}(\Delta), B_{i}^{*} \cap h_{t}(\Delta)\right) \mid i \in \Lambda\right\}$ is an essential family for $h_{t}(\Delta)$. Let $M_{0}=\pi_{k}\left(h_{t}(\Delta)\right.$. By the reasoning just used, $\left\{\left(A_{i}^{*} \cap M_{0}, B_{i}^{*} \cap M_{0}\right) \mid i \in \Lambda\right\}$ is an essential family for $M_{0}$, and since $h_{t}(\Delta) \subset K \subset V, M_{0} \subset U$.

Now note that

$$
\begin{aligned}
M_{0} & \subset \pi_{k}\left(\cap\left\{\tilde{L}_{j} \mid j \in \Lambda_{1} \cup \Lambda_{2}\right\}\right)=\pi_{k}\left(\bigcap\left\{L_{j}^{*} \times Q_{k} \mid j \in \Lambda_{1} \cup \Lambda_{2}\right\}\right) \\
& =\pi_{k}\left(M \times Q_{k}\right)=M .
\end{aligned}
$$

But for each $x \in T, M_{0}$ contains at most one point of $\pi_{1}^{-1}(x) \cap I^{k}$, so $M_{0}$ is totally disconnected. Hence we see that $\operatorname{dim} M_{0}<\operatorname{dim} M=m+2$. But since card $\Lambda=m$ +1 , then $\operatorname{dim} M_{0}=m+1$. Now we have $F \mid M_{0}: M_{0} \rightarrow K_{m}$. Reviewing Lemma 2.1, we see that the existence of the map $F \mid M_{0}$ leads to a contradiction. This completes the proof of 4.1 .

4.3. Generalization. In [Wa1] there was another theorem, Theorem 3.2, in which it was shown that certain spaces have infinite cohomological dimension. The difference is that in 3.2 , the space is the intersection, in a special way, of continuumwise separators. The way the result is stated, these continuum-wise separators do not necessarily extend to continuum-wise separators of the appropriate faces of the Hilbert cube. Because of this, we do not know how to apply our technique of proof to a case in which the hypotheses are parallel to those in 3.2 of [Wa1]. On the other hand, if we change separator to continuum-wise separator in 3.2(b), then Theorem 4.1 is still true, and in some sense the second theorem of [Wa1] is generalized. To prove the result, only minor changes in the proof of 4.1 are needed. Using 4.4 below, you may assume that for $j \in \Lambda_{1}, L_{j}$ is a separator instead of continuum-wise separator. In place of 5.5 and 5.2, use 5.6 and 5.2 of [RSW] to ascertain that $K$ contains a continuum from $A_{1}$ to $B_{1}$.

4.4. Lemma. Let $L$ be compact and $A, B$ be disjoint closed subsets of $L$. Let $L_{0}$ be a continuum-wise separator of $(A, B)$ in $L$ and let $U$ be a neighborhood of $L_{0}$. Then there exists a separator $S$ of $(A, B)$ in $L$ such that $S \subset U$.

This is Proposition 5.2 of [Bo]. 


\section{REFERENCES}

[Bo] P. L. Bowers, Detecting cohomologically stable mappings, Proc. Amer. Math. Soc. 86 (1982), 679-684.

[GS] Dennis J. Garity and Richard M. Schori, Infinite-dimensional dimension theory, preprint.

[Po1] Roman Pol, Countable dimensional universal sets, preprint.

[Ru1] Leonard R. Rubin, Hereditarily strongly infinite dimensional spaces, Michigan Math. J. 27 (1980), 65-73.

[Ru2] 157-166.

[Ru3] , Totally disconnected spaces and infinite cohomological dimension, Topology Proc. 7 (1982), More compacta of infinite cohomological dimension, Contemp. Math., vol. 44, Amer. Math. Soc., Providence, R. I., 1985, pp. 221-226.

[Ru4] , Cell-like maps, dimension and cohomological dimension: a survey, Geometric and Algebraic Topology, Banach Center Publications, Volume 18, PWN-Polish Scientific Publishers, Warsaw, 1986, pp. 397-402.

[RSW] Leonard R. Rubin, R. M. Schori, and J. J. Walsh, New dimension theory techniques for constructing infinite-dimensional examples, General Topology Appl. 10 (1979), 83-102.

[Wa1] John J. Walsh, A class of spaces with infinite cohomological dimension, Michigan Math. J. 27 (1980), 215-222.

[Wa2] _ Dimension, cohomological dimension, and cell-like mappings, Shape Theory and Geometric Topology, Proceedings, Dubrovnik 1981, Lecture Notes in Math., vol. 870, Springer-Verlag, Berlin and New York, 1981, pp. 105-118.

Department of Mathematics, University of Zagreb, Zagreb, Yugoslavia

Department of Mathematics, University of OKLAhoma, Norman, OKLAhOMa 73019 